\title{
Pure Non-Blocking LC Based Matrix Optical Switch for All-Optical Fibre Networks
}

\author{
Yuri Panarin \\ Technological University Dublin, yuri.panarin@tudublin.ie \\ V. Alex \\ Technological University Dublin
}

Follow this and additional works at: https://arrow.tudublin.ie/engscheleart2

Part of the Electrical and Computer Engineering Commons

\section{Recommended Citation}

Panarin, Yu. P. (2007). Pure non-blocking LC based matrix optical switch for all-optical fibre networks. Molecular Crystals and Liquid Crystals , vol. 476, pp. 95/[341] - 104/[350].

This Article is brought to you for free and open access by the School of Electrical and Electronic Engineering at ARROW@TU Dublin. It has been accepted for inclusion in Articles by an authorized administrator of ARROW@TU Dublin. For more information, please contact arrow.admin@tudublin.ie, aisling.coyne@tudublin.ie, gerard.connolly@tudublin.ie.

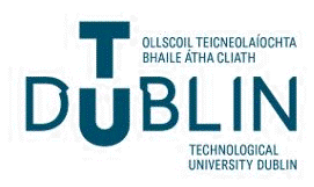




\title{
Pure Non-Blocking LC Based Matrix Optical Switch for All-Optical Fibre Networks
}

\author{
Yu. P. Panarin \\ V. Alex \\ School of Electronic \& Communication Engineering, Dublin Institute \\ of Technology, Dublin, Ireland
}

New pure non-blocking matrix optical switch scheme for fiber networks is presented. The optical switch is based on conventional LCD technology, where the each pixel controls the polarization state of the light beam. The suggested switch offers several advantages over the conventional cross-point architecture such as: cost; complexity; size; adjustment; and optical performance

Keywords: electro-optics; fiber-optic communications; liquid crystals; optical switching

\section{INTRODUCTION}

Explosive growth in data communications is changing the requirements for the networks, demanding for wider and wider bandwidth. At present, more and more communication transmission infrastructures are based on optical fibre links with practically unlimited bandwidth. The core part of any optical fibre network is an optical switch that allows to route signals between different terminals. This demand is encouraging a new generation of technologies designed to make networks faster, more reliable and more scalable.

The optical switches can be classified into two categories. The first category changes the optical signal into electrical and performs switching function before changing it back to optical. Such an optical-electrical-optical (OEO) switch, or opaque switch, provides a very high degree of flexibility. On the other hand, the opaque switches,

Address correspondence to Yu. P. Panarin, School of Electronic \& Communication Engineering, Dublin Institute of Technology, Kevin Street, Dublin 8, Ireland. E-mail: yuri.panarin@dit.ie 
have an increasing cost per port as bandwidth increases and could become high cost centers in the future network.

The second category is a pure optical (or Photonic) switch where optical-electrical-optical conversion is not necessary. Photonic switches have a fixed cost per port regardless of the amount of bandwidth through each port (or wavelength in WDM systems), because they switch light, i.e., they are bit-rate independent. For this reason, at very high bandwidths, the cost of photonic switching is very attractive compared to opaque switches. Since the bandwidth per port is virtually unlimited by today's standards, a single switch can allow scalability into the hundreds of terabits per second, allowing extremely high nodal scalability. There are a number of photonic switching technologies, including: Micro-Electro-Mechanical Systems (MEMS), Thermo-optics (e.g., bubble) technology, Liquid Crystal switching, etc... Currently the optical switching is performed by Micro Electro-Mechanical Switches (MEMS), which are based on mechanical movements of the mirrors. This limits the switching speed and lifetime of such optical switches.

On the other hand, the optical switching can be easily achieved with help of Liquid Crystals, utilizing a number of different electro-optic effects such as: TN; total reflection [1]; FLC [2]; holographic; cholesteric mirrors [3]; etc ... Although binary (1-to-2 or 2-to-2) LC based optical switches are well known, the larger (matrix) optical switches are not yet commercialised. The main reason is follows. In conventional matrix approach to perform non-blocking $\mathrm{N}$ to $\mathrm{N}$ switching it is necessary to use a matrix with $\mathrm{N} \times \mathrm{N}$ single Switching Elements (SE), each placed on the cross-point of input and output channels. This cross-point architecture offers simple control and wide-sense non-blocking switching which allows routing of any input to any unused output without disturbing other connections. Nevertheless this approach has also several key drawbacks:

- Large number of single switches $\mathrm{S}=\mathrm{N}^{2}$. This dramatically increases the total cost of the matrix and complicates its adjustment and reliability.

- The light beam is travelling through $\mathrm{N}$ switches accumulating extra insertion loss and cross-talk. In other words, even if the single switch's performance is rather fascinating - the total matrix switch performance will be unacceptable.

The last statement can be illustrated by the following example. Let us consider cross-point $\mathrm{N} \times \mathrm{N}$ ports optical switch and the contrast ratio of the single switch $\mathrm{K}=1000$, which corresponds to cross-talk $\mathrm{CT}_{1}=10 \log (1 / \mathrm{K})=-30 \mathrm{~dB}$. This means that about $0.1 \%$ of the input power flows in the wrong direction. Although such a small amount 
residual (strain) light does not affect much the power in the output channel (or insertion losses) but it significantly worsens the overall cross-talk ratio of the proposed matrix switch.

The overall crosstalk $\mathrm{N} \times \mathrm{N}$ ports matrix switch is

$$
C T_{N}=10 \cdot \log (N-1)+C T_{1}
$$

Therefore for practical 16- and 64-ports switches the final crosstalk will be $\mathrm{CT}_{16}=-18.24 \mathrm{~dB}$ and $\mathrm{CT}_{64}=-12 \mathrm{~dB}$ correspondingly.

Recently we have suggested and patented new approach for LC based multi-channel matrix optical switch. The main idea of this scheme is a use of conventional LCD technology to perform parallel switching in all optical channels.

\section{BASIC SWITCH DESCRIPTION}

\section{Binary Switching Element}

The core element of the proposed scheme is TN LC cell in conjunction with lateral displacement beamsplitter (LBS) as shown in the Figure 1.

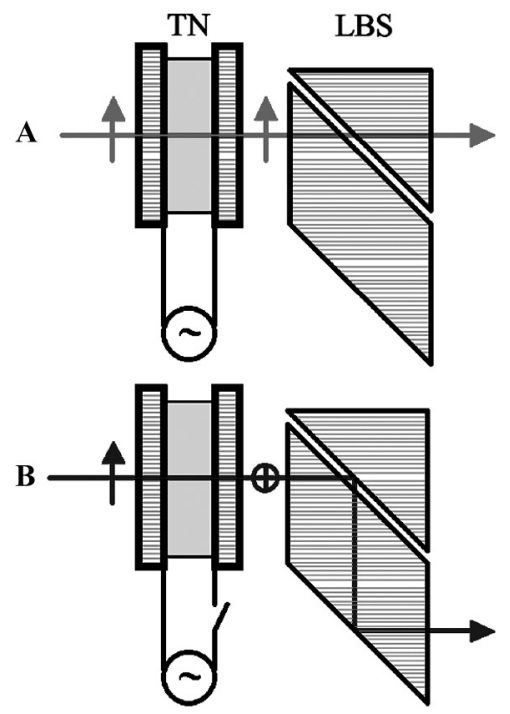

(a)
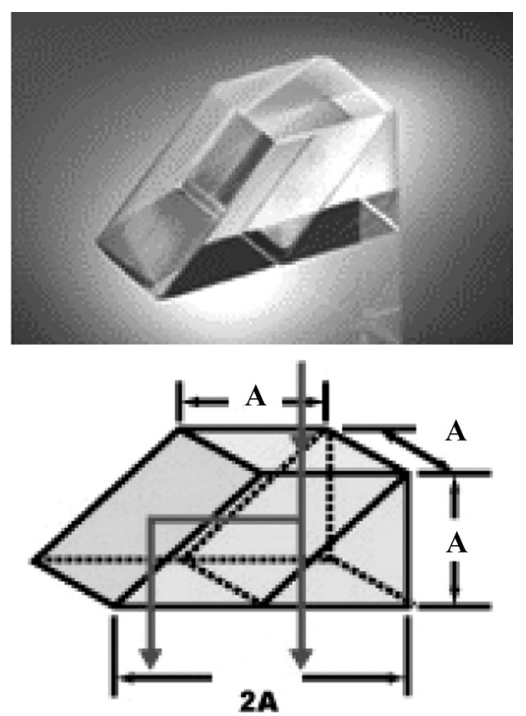

(b)

FIGURE 1 (a) Schematic diagram of bypass/shift optical switch based on TN cell and (b) TECH SPEC ${ }^{\mathrm{TM}}$ Lateral Displacement Beamsplitters (http:// www.edmundoptics.com/WE//). 
When the voltage is applied to LC pixel (A case) the light beam passes through the LC layer (pixel) without changing the direction of polarization - this corresponds to "bypass" state. When there is no voltage on the pixel (B case), the pixel is in twisted nematic (TN) state and rotates the polarization by $90^{\circ}$ to perform lateral displacement. Therefore such switch performs simple 1-to-2 binary switching.

In matrix optical switch we suggested to use a simple and inexpensive LC matrix (LCM) in conjunction with lateral displacement beamsplitter (LBS) as a core part of Switching Array (SA). Such LCM, performs parallel and independent switching of all input optical channels (N-to-2N), where one pixel of the matrix performs 1-to-2 binary switching.

\section{Basic Matrix Switch}

The architecture and performance of proposed switch in simplest (basic) $4 \times 4$ port matrix switch is shown in the Figure 2 . The matrix optical switch can be assembled by simple stacking two SAs the first of them performs horizontal (right) shift on 2-pixel distance and the second one - vertical (down) shift; the birefringent film; four squareshaped collector lenses and four output fibres.

LCM consists of $16(4 \times 4)$ pixels which are arranged in square fashion in four 4-pixels groups (Fig. 2a). Each pixel has 4-digit code (index): $k l m n$ where $k l$ defines the index of the group and $m n$ defines the index within each group. All the input channels are coming to the first $(k l=00)$ group and the index of channel is a binary number of $m n$ (e.g., $2 m+n$ ). The output channels collect all traces from the proper groups and the index of output channel is a binary number of $k l$ (e.g., $2 k+l$ ).

\begin{tabular}{|c|c|c|c|}
\hline 0000 & 0001 & 0100 & 0101 \\
\hline 0010 & 0011 & 0110 & 0111 \\
\hline 1000 & 1001 & 1100 & 1101 \\
\hline 1010 & 1011 & 1110 & 1111 \\
\hline
\end{tabular}

(a)

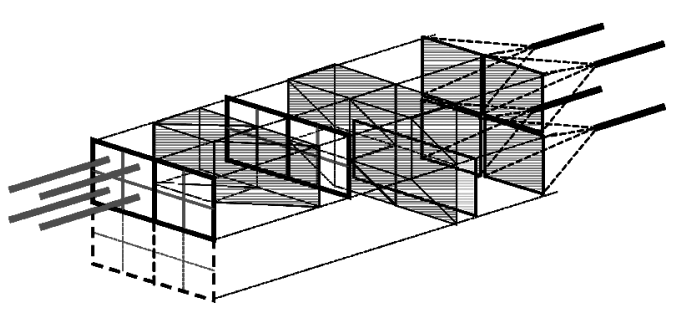

(b)

FIGURE 2 (a) Spatial distribution of input optical routs and (b) total construction of $4 \times 4$ matrix switch. 


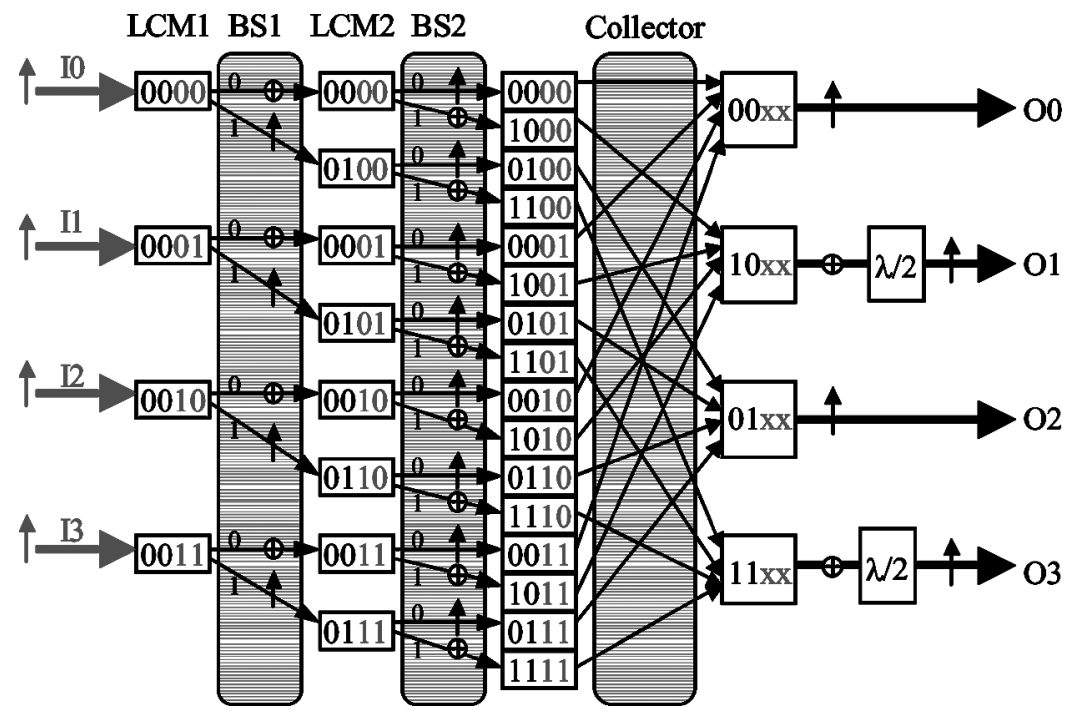

FIGURE 3 Switching network for non-blocking $4 \times 4$ channels optical switch.

Such indexing provides simple routing algorithm e.g. $k \neq m$ is a condition for horizontal switching and $l \neq m$ - for vertical switching. Horizontal switching (shift) occurs when the light polarisation is vertical $(\uparrow)$ and vertical switching - for horizontal polarization $(+)$. The TN LC pixel rotates polarisation if there is no voltage on it (0) and leaves polarization without change if voltage is applied (1). The complete switching network is shown in the Figure 3.

The final polarization of the output channels is vertical if $\mathrm{k}=0$ and horizontal if $\mathrm{k}=1$. If necessary the output polarisation of the $\mathrm{k}=1$ channels can be easily corrected (i.e., changed to the vertical) by introduction the half-wave $(\lambda / 2)$ birefringent plate (film) or extra TN LCM. The actual position of this film is on the rear side, bottom half of the second (last) beamsplitters (Fig. 2b).

\section{LARGER OPTICAL SWITCHES}

The architecture of larger optical switches is similar to the described $4 \times 4$ switch $(\mathrm{N}=4, \mathrm{n}=2)$ with only one important difference - it needs extra pair of beamsplitters with different (double) lateral displacement. Lets consider larger $16 \times 16$ optical switch $(\mathrm{N}=16, \mathrm{n}=4$ and $\left.\mathrm{N}=\mathrm{n}^{2}\right)$. In this case the LCM consists of $256(16 \times 16)$ pixels as shown in the Figure 4. 


\begin{tabular}{||l|l|l|l||l|l|l|l||l|l|l|l|||l|l|l|l||}
\hline 0000 & 0001 & 0002 & 0003 & 0100 & 0101 & 0102 & 0103 & 0200 & 0201 & 0202 & 0203 & 0300 & 0301 & 0302 & 0303 \\
\hline 0010 & 0011 & 0012 & 0013 & 0110 & 0111 & 0112 & 0113 & 0210 & 0211 & 0212 & 0213 & 0310 & 0311 & 0312 & 0313 \\
\hline 0020 & 0021 & 0022 & 0023 & 0120 & 0121 & 0122 & 0123 & 0220 & 0221 & 0222 & 0223 & 0320 & 0321 & 0322 & 0323 \\
\hline 0030 & 0031 & 0032 & 0033 & 0130 & 0131 & 0132 & 0133 & 0230 & 0231 & 0232 & 0233 & 0330 & 0331 & 0332 & 0333 \\
\hline \hline 1000 & 1001 & 1002 & 1003 & 1100 & 1101 & 1102 & 1103 & 1200 & 1201 & 1202 & 1203 & 1300 & 1301 & 1302 & 1303 \\
\hline 1010 & 1011 & 1012 & 1013 & 1110 & 1111 & 1112 & 1113 & 1210 & 1211 & 1212 & 1213 & 1310 & 1311 & 1312 & 1313 \\
\hline 1020 & 1021 & 1022 & 1023 & 1120 & 1121 & 1122 & 1123 & 1220 & 1221 & 1222 & 1223 & 1320 & 1321 & 1322 & 1323 \\
\hline 1030 & 1031 & 1032 & 1033 & 1130 & 1131 & 1132 & 1133 & 1230 & 1231 & 1232 & 1233 & 1330 & 1331 & 1332 & 1333 \\
\hline \hline 2000 & 2001 & 2002 & 2003 & 2100 & 2101 & 2102 & 2103 & 2200 & 2201 & 2202 & 2203 & 2300 & 2301 & 2302 & 2303 \\
\hline 2010 & 2011 & 2012 & 2013 & 2110 & 2111 & 2112 & 2113 & 2210 & 2211 & 2212 & 2213 & 2310 & 2311 & 2312 & 2313 \\
\hline 2020 & 2021 & 2022 & 2023 & 2120 & 2121 & 2122 & 2123 & 2220 & 2221 & 2222 & 2223 & 2320 & 2321 & 2322 & 2323 \\
\hline 2030 & 2031 & 2032 & 2033 & 2130 & 2131 & 2132 & 2133 & 2230 & 2231 & 2232 & 2233 & 2330 & 2331 & 2332 & 2333 \\
\hline \hline 3000 & 3001 & 3002 & 3003 & 3100 & 3101 & 3102 & 3103 & 3200 & 3201 & 3202 & 3203 & 3300 & 3301 & 3302 & 3303 \\
\hline 3010 & 3011 & 3012 & 3013 & 3110 & 3111 & 3112 & 3113 & 3210 & 3211 & 3212 & 3213 & 3310 & 3311 & 3312 & 3313 \\
\hline 3020 & 3021 & 3022 & 3023 & 3120 & 3121 & 3122 & 3123 & 3220 & 3221 & 3222 & 3223 & 3320 & 3321 & 3322 & 3323 \\
\hline 3030 & 3031 & 3032 & 3033 & 3130 & 3131 & 3132 & 3133 & 3230 & 3231 & 3232 & 3233 & 3330 & 3331 & 3332 & 3333 \\
\hline \hline
\end{tabular}

FIGURE $416 \times 16$-pixel LC matrix. Pixel Index: klmn. Input Channels: IO $-I 15(I=4 m+n)$. Output Channels: OO -O15 $(O=4 k+l)$.

The pixels are again arranged in square fashion in sixteen 16-pixels $(4 \times 4)$ groups. The pixels indices are the same as for the described switch. The basic beamsplitter shifts a beam on 4 pixels (i.e., one group) down/right. To address the first input channel $I 0$ to the output O15 it is necessary to use 6 such beamsplitters: three for horizontal shift and other three - for vertical. In general, number of LCMs (M) $\mathrm{M}=2 \cdot(\mathrm{n}-1)$. Nevertheless, there is a way to decrease the total number of LCMs and beamsplitters. This can be done by two different pairs of beamsplitters: first (basic) pair (BSH1 and BSV1) shifts on 4 pixels (i.e., one group) and the second is pair of "double strength" BS (BSH2 and BSV2) which shifts on 8 pixels (i.e., two groups). The construction of $16 \times 16$ optical switch is shown in the Figure 5 .

The switching network for non-blocking $16 \times 16$ channels optical switch is shown in the Figure 6 . Similar to $4 \times 4$ switch, the final

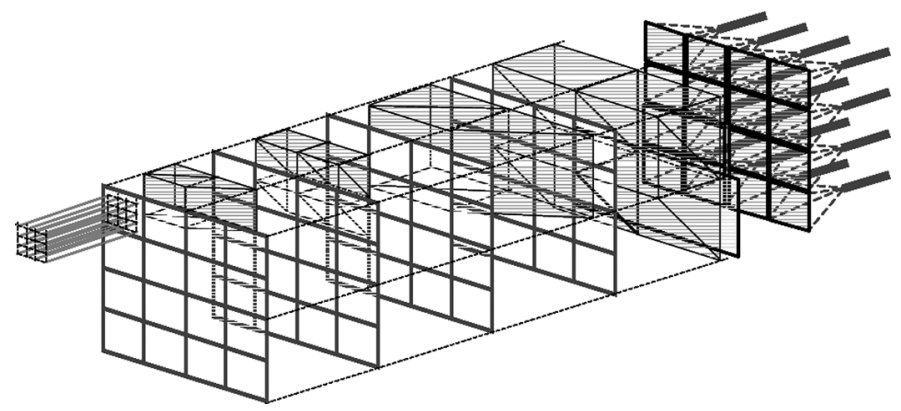

FIGURE 5 The construction of $16 \times 16$-ports LC matrix switch. 


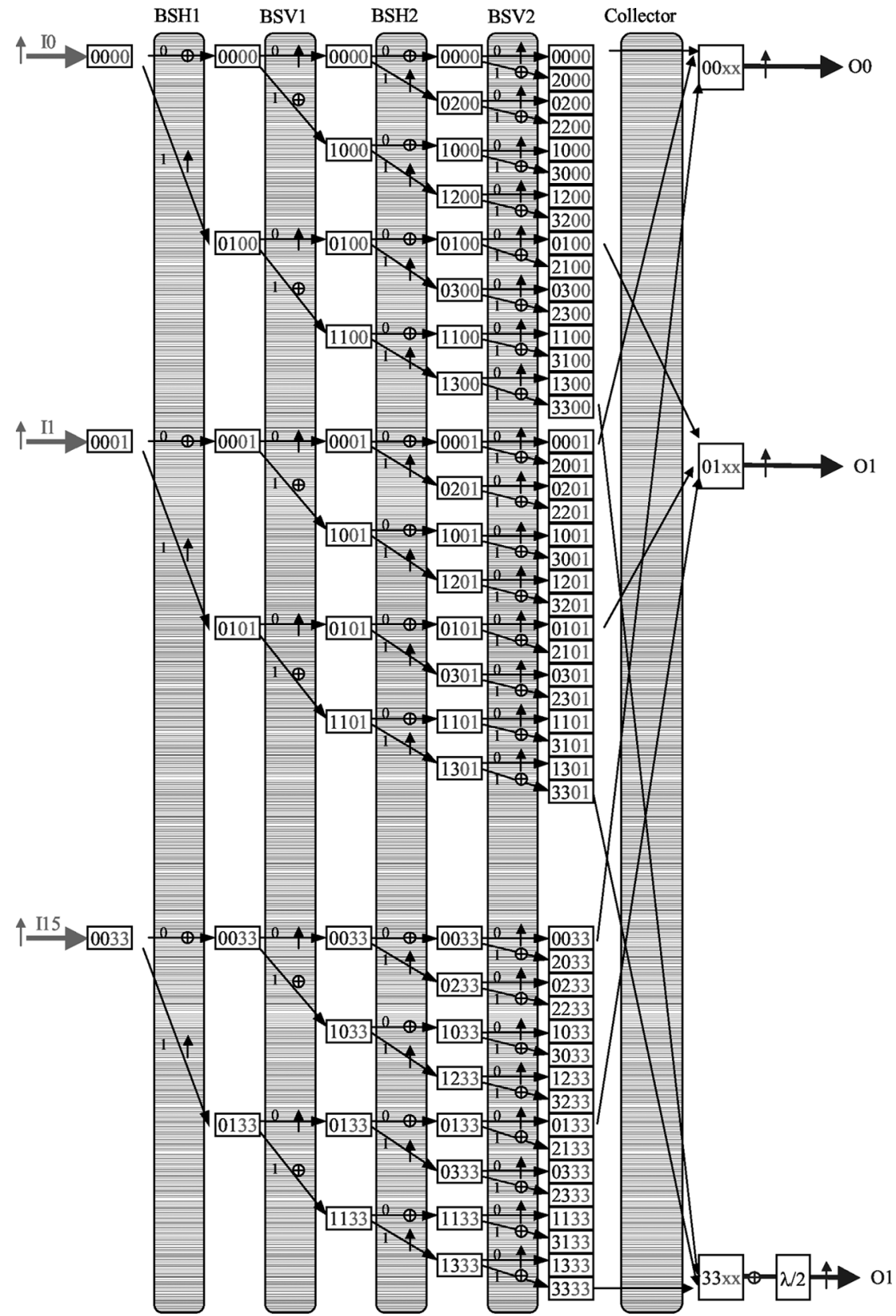

FIGURE 6 Switching network for non-blocking $16 \times 16$ channels optical switch. 
polarization of the output channels with $\mathrm{k}=0$ or 1 is vertical and $\mathrm{k}=2,3$ is horizontal. The output polarisation of the $\mathrm{k}=2,3$ channels can be easily corrected by placing the half-wave $(\lambda / 2)$ birefringent plate (film) on the rear side, bottom half of the forth (last) beamsplitter.

Similarly larger $64 \times 64$ optical switch $(\mathrm{N}=64, \mathrm{n}=8)$ consists of 3 different pairs of beamsplitters: first (basic) pair (BSH1 and BSV1) shifts on 8 pixels (i.e., one group), the second is pair of "double strength" BS (BSH2 and BSV2) which shifts on 16 pixels (i.e., two groups) and the third is a pair of "quarto-strength" BS (BSH4 and BSV4) which shifts on 32 pixels (i.e., four groups). In this scheme $\mathrm{n}(\mathrm{n}=2,4$, $8, \ldots)$ is a power of $2^{\mathrm{q}}(\mathrm{q}=1,2,3)$ and the total number of SAs is

$$
M=2 \cdot \log _{2}(\sqrt{N})=2 \cdot \log _{2}(n)=2 \cdot q .
$$

\section{FURTHER IMPROVEMENTS}

The proposed switching scheme possesses some features and peculiarities enabling further useful modifications.

LCM reduction. In matrix switch the "active" or switching area of LCM depends on the switching stage, i.e., for the first stage it is $\mathrm{N}$ $(\mathrm{n} \times \mathrm{n})$-pixels group of whole $\mathrm{N} \times \mathrm{N}$-pixels matrix with index $00 \mathrm{xx}$ (see Fig. 2). The active area of the second stage consists of two groups with the indices $00 \mathrm{xx}$ and $01 \mathrm{xx}$. In general, in $\mathrm{N} \times \mathrm{N}$ ports matrix switch the active area of the last stage is just upper half of $\mathrm{N} \times \mathrm{N}$ pixels LCM. Therefore $\mathrm{N} \times \mathrm{N}$ ports matrix switch needs $\mathrm{N}$-columns by $\mathrm{N} / 2$ rows LCMs.

Mosaic addressing. In general, $\mathrm{N} \times \mathrm{N}$ ports matrix switch consists of $2 q \mathrm{~N} \times \mathrm{N} / 2$-pixels (or $\mathrm{N}^{2} / 2$ ) LCMs. Therefore for practical matrix switches (16 or 64-ports) the total number of pixels becomes rather large and LCM needs matrix addressing rather than simplest mosaic addressing. The passive-matrix addressing reduces the quality (i.e., cross-talk) of the optical switching, while active-matrix addressing increases the cost of LCM. This drawback can be cured by driving the pixels with indices xxmn (e.g., 0000, 0100, 1000 and 1100, see Fig. 2a) by the same control line, i.e., connecting these pixels to the same control line. In this case the number of independent control lines reduces form $\mathrm{N}^{2} / 2$ to just $\mathrm{N}$ enabling simple and inexpensive mosaic addressing.

If necessary the simplest TN LCMs can be replaced by much faster FLC matrices or higher quality vertical aligned (VA) LCMs or other LC based switches. 


\section{THE CROSS-TALK CLEANING}

It was mentioned that the overall matrix switch crosstalk is worse than the crosstalk of the single switch and depends on the number of stages that the beams pass through (Eq. (1)) and becomes unacceptably low in cross-point matrix switch. The suggested matrix switch offers much better overall crosstalk, due to lower number of switching stages (compare $\mathrm{M}$ and N, Eq. (2))

In general, assuming that $C T_{1}$ is a cross-talk of the single switch (stage), the overall crosstalk of the $\mathrm{N}$-channels optical switch $\left(C T_{N}\right)$ is:

$$
\begin{aligned}
C T_{N}= & 10 \cdot \log (\sqrt{N-1})+C T_{1} \approx 10 \cdot \log (n) \\
& +C T_{1}=10 \cdot \log _{2}(n) / 3.32+C T_{1} \approx 3 q-C T_{1}
\end{aligned}
$$

Thus the overall crosstalk of 64 -channels switch $(\mathrm{N}=64, \mathrm{n}=8, \mathrm{q}=3)$ will be about $-21 \mathrm{~dB}$, which is rather low for practical application.

It is possible to eliminate the parasitic effect of all the residual light beams by introducing the cross-talk removal polarizer between the final (last) SA and collectors as shown in the Figure 7. Let us consider

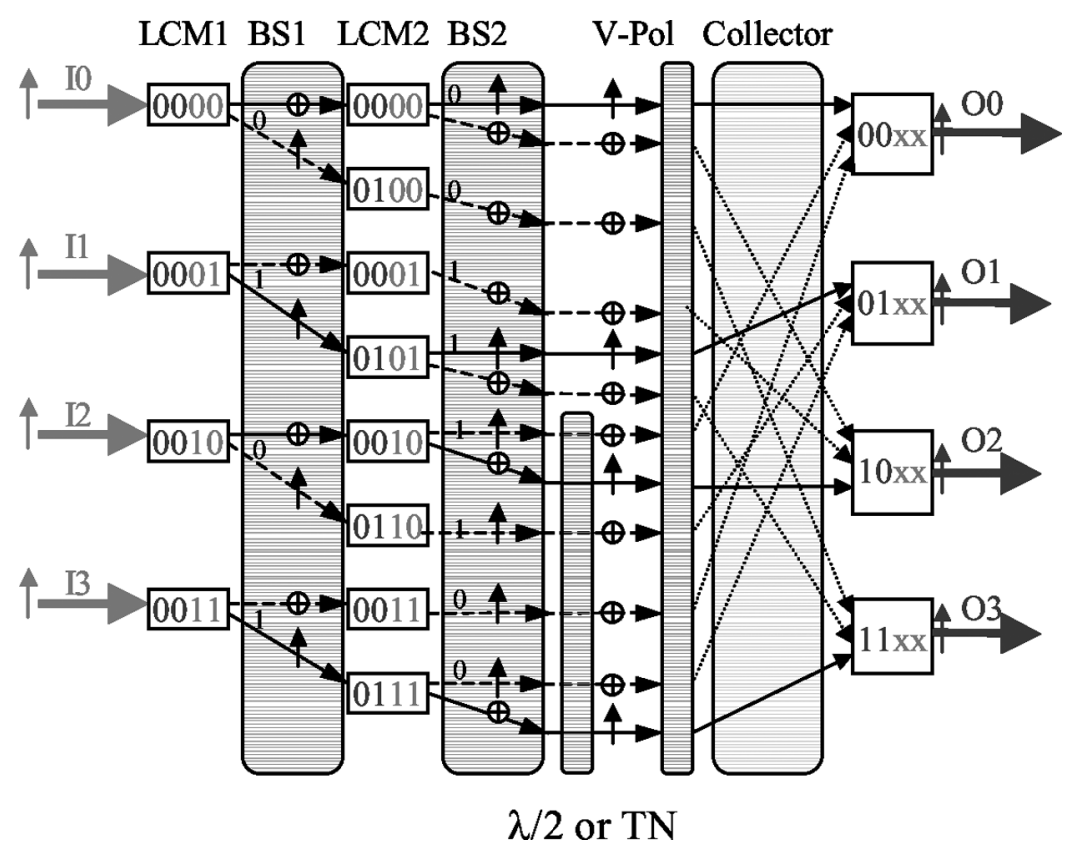

FIGURE 7 Polarization states and crosstalk cleaning in blocking $4 \times 4$ channels optical switch. 
that 4-ports switch is configured to connect the input ports to the output ports with the same number, i.e. I0 to O0, I1 to O1, etc. The main beam traces are drawn as solid lines (arrows), while the residuals beams as dashed lines. It is interesting to find (see Fig. 7) that all the main beams in upper half of the switch are vertically polarised, while all the residual beams are horizontally polarised. The opposite is valid for the bottom half of the switch. After passing through the $\lambda / 2$ (or TN) layer the polarisations of all main beams will change to the vertical and all residual beams to horizontal. If the axis of this polarizer is vertical, all the residual channels will not pass through it to the collectors. Therefore the overall crosstalk will be as good as (or even better) than for individual optical switch.

\section{CONCLUSION}

In present article we described only the principles of new approach for LC based matrix optical switch, which shows several advantages over the conventional cross-point architecture such as: cost; complexity; size; adjustment; and optical performance. The practical design/ application of this scheme requires further research and development of other important aspects/ components, which were not considered in present article, such as collector lenses, fiber coupling, polarizers, LCMs, etc.

\section{REFERENCES}

[1] Soref, R. A. (1979). Opt. Lett., 4, 155.

[2] Meadows, M. R., Handschy, M. A., \& Clark, N. A. (1989). Appl. Phys. Lett., 54, 1394.

[3] Semenova, Yu., Panarin, Yu., Dovgalets, S., \& Farrell, G. (2004). Mol. Cryst. Liq. Cryst., 413, 385. 